\section{On Input-to-State Stability of Stochastic Retarded Systems With Markovian Switching}

\author{
Lirong Huang and Xuerong Mao
}

\begin{abstract}
This note develops a Razumikhin-type theorem on $p$ th moment input-to-state stability of hybrid stochastic retarded systems (also known as stochastic retarded systems with Markovian switching), which is an improvement of an existing result. An application to hybrid stochastic delay systems verifies the effectiveness of the improved result.
\end{abstract}

Index Terms-Input-to-state stability (ISS), Markov chain, Razumikhintype theorems, stochastic systems, time delay.

\section{INTRODUCTION}

Since Markov jump linear systems were firstly introduced in early 1960s (see, e.g., [16] and [25]), the hybrid systems driven by continuous-time Markov chains have been widely employed to model many practical systems where they may experience abrupt changes in system structure and parameters such as failure prone manufacturing, power systems, solar-powered systems and battle management in command, control and communication systems (see [1], [4], [14], [16], [21], and references therein). An area of particular interest has been the stability analysis of this class of hybrid systems and its applications to automatic control (see, e.g., [3], [8], and [16]). When time delays and environmental noise are taken into account, which are often encounterd in real systems and may be the cause of poor performance and instability, the hybrid systems are described with stochastic functional differential equations with Markovian switching and called hybrid stochastic retarded systems (HSRSs). One of the most important HSRSs that frequently appear in engineering is those called hybrid stochastic delay systems (HSDSs), which are also known as stochastic delay systems with Markovian switching (SDSwMS) and described with stochastic differential delay equations with Markovian switching (see, e.g., [14], [15] and [24]).

Recently, hybrid stochastic retarded systems (HSRSs) have been widely used since stochastic modelling plays an important role in many branches of science and engineering. Consequently, stability analysis of HSRSs and HSDSs has been studied by many works, see, e.g., [7], [13]-[15], [24], and [25]. Among the key results, Mao et al. ([15]) and Huang et al. ([7]) proposed the Razumikhin-type theorems on stability of hybrid stochastic retarded systems and their applications to hybrid stochastic delay systems. The Razumikhin method is developed to cope with the difficulty arisen from the large, fast varying and nondifferentiable time delays (see, e.g., [14] and [15]). The Razumikhin-type techniques have been applied to establish stability criteria for stochastic functional differential equations (see [12] and [6]). Since the results for non-switched systems cannot be simply extended to systems with jumps and switching (see, e.g., [8] and [16]), Razumikhin-type Theorems for HSRSs and their applications are developed in [15] and [7]. However, some conditions of results in [15] and [7] may be too conservative. This note is to improve the

Manuscript received January 22, 2009; revised March 17, 2009. First published July 24, 2009; current version published August 05, 2009. This work was supported by the UK ORSAS and University of Strathclyde. Recommended by Associate Editor Z. Wang.

The authors are with the Department of Mathematics and Statistics, University of Strathclyde, Glasgow G1 1XH, U.K. (e-mail: lirong@ @stams.strath.ac.u; xuerong@stams.strath.ac.uk).

Digital Object Identifier 10.1109/TAC.2009.2022112
Razumikhin-type theorem proposed in [7] and make it more applicable (see Remark 3.2 and Example 4.1).

\section{NOTATION}

Throughout the note, unless otherwise specified, we shall employ the following notation. Let $\left(\Omega, \mathcal{F},\left\{\mathcal{F}_{t}\right\}_{t>0}, \mathbb{P}\right)$ be a complete probability space with a filtration $\left\{\mathcal{F}_{t}\right\}_{t \geq 0}$ satisfying the usual conditions (i.e., it is right continuous and $\mathcal{F}_{0}$ contains all $\mathbb{P}$-null sets) and $\mathbb{E}[\cdot]$ be the expectation operator with respect to the probability measure. Let $B(t)=\left(B_{1}(t), \ldots, B_{m}(t)\right)^{T}$ be an $m$-dimensional Brownian motion defined on the probability space. If $x, y$ are real numbers, then $x \vee y$ denotes the maximum of $x$ and $y$, and $x \wedge y$ stands for the minimum of $x$ and $y$. Let $|\cdot|$ denote the Euclidean norm in $R^{n}$. Let $\tau \geq 0$ and $C\left([-\tau, 0] ; R^{n}\right)$ denote the family of all continuous $R^{n}$-valued functions $\varphi$ on $[-\tau, 0]$ with the norm $\|\varphi\|=\sup \{|\varphi(\theta)|:-\tau \leq \theta \leq 0\}$. Let $C_{\mathcal{F}_{0}}^{b}\left([-\tau, 0] ; R^{n}\right)$ be the family of all $\mathcal{F}_{0}$-measurable bounded $C\left([-\tau, 0] ; R^{n}\right)$-valued random variables $\xi=\{\xi(\theta):-\tau \leq \theta \leq 0\}$. For $p>0$ and $t \geq 0$, denote by $L_{\mathcal{F}_{t}}^{p}\left([-\tau, 0] ; R^{n}\right)$ the family of all $\mathcal{F}_{t}$-measurable $C\left([-\tau, 0] ; R^{n}\right)$-valued random processes $\phi=\{\phi(\theta)$ : $-\tau \leq \theta \leq 0\}$ such that $\sup _{-\tau \leq \theta \leq 0} \mathbb{E}|\phi(\theta)|^{p}<\infty$. We let $\mathcal{K}$ denote the class of continuous strictly increasing functions $\mu$ from $R_{+}$ to $R_{+}$with $\mu(0)=0$. Let $\mathcal{K}_{\infty}$ denote the class of functions $\mu \in \mathcal{K}$ with $\mu(r) \rightarrow \infty$ as $r \rightarrow \infty$. Functions in $\mathcal{K}$ and $\mathcal{K}_{\infty}$ are called class $\mathcal{K}$ and $\mathcal{K}_{\infty}$ functions, respectively. If $\mu \in \mathcal{K}$, its inverse function is denoted by $\mu^{-1}$ with domain $[0, \mu(\infty))$. We denote by $\mu \in V \mathcal{K}$ and $\mu \in C \mathcal{K}$ if $\mu \in \mathcal{K}$ and $\mu$ is convex and concave, respectively. In this note, a function $\beta: R_{+} \times R_{+} \rightarrow R_{+}$is said to be of class $\mathcal{K} \mathcal{L}$ if for each fixed $t$ the mapping $\beta(\cdot, t)$ is of class $\mathcal{K}$ and for each fixed $s$ the function $\beta(s, t)$ is decreasing to zero on $t$ as $t \rightarrow \infty$. We also let $\mathcal{L}_{\infty}^{l}$ denote the class of essentially bounded functions $u: R_{+} \rightarrow R^{l}$ with $\|u\|_{\infty}=\operatorname{ess~sup}_{t>0}|u(t)|<\infty$.

Let $r(t), t \geq 0$, be a right-continuous Markov chain on the probability space taking values in a finite state space $S=\{1,2, \ldots, N\}$ with generator $\Gamma=\left(\gamma_{i j}\right)_{N \times N}$ given by

$$
P\{r(t+\Delta)=j: r(t)=i\}= \begin{cases}\gamma_{i j} \Delta+o(\Delta) & \text { if } i \neq j \\ 1+\gamma_{i i} \Delta+o(\Delta) & \text { if } i=j\end{cases}
$$

where $\Delta>0$ and $\gamma_{i j} \geq 0$ is the transition rate from $i$ to $j$ if $i \neq j$ while $\gamma_{i i}=-\sum_{j \neq i} \gamma_{i j}$. Assume that the Markov chain $r(\cdot)$ is independent of the Brownian motion $B(\cdot)$. It is known that almost all sample paths of $r(t)$ are right-continuous step functions with a finite number of simple jumps in any finite subinterval of $R_{+}:=[0, \infty)$.

Let us consider an $n$-dimensional HSRS

$$
\mathrm{d} x(t)=f\left(x_{t}, t, r(t), u_{d}(t)\right) \mathrm{d} t+g\left(x_{t}, t, r(t), u_{d}(t)\right) \mathrm{d} B(t)
$$

on $t \geq 0$ with initial data $x_{0}=\{x(\theta):-\tau \leq \theta \leq 0\}=\xi \in$ $C_{\mathcal{F}_{0}}^{b}\left([-\tau, 0] ; R^{n}\right)$ and $r(0)=r_{0} \in S$, where $x_{t}=\{x(t+\theta):-\tau \leq$ $\theta \leq 0\}$ is regarded as a $C\left([-\tau, 0] ; R^{n}\right)$-valued random variable and $u_{d} \in \mathcal{L}_{\infty}^{l}$ the disturbance input. Moreover, $f: C\left([-\tau, 0] ; R^{n}\right) \times R_{+} \times$ $S \times R^{l} \rightarrow R^{n}$ and $g: C\left([-\tau, 0] ; R^{n}\right) \times R_{+} \times S \times R^{l} \rightarrow R^{n \times m}$ are measurable functions with $f(0, t, i, 0) \equiv 0$ and $g(0, t, i, 0) \equiv 0$ for all $t \geq 0$. So (1) admits a trivial solution $x(t ; 0) \equiv 0$. We assume that $f$ and $g$ are sufficiently smooth so that (1) has a unique solution on $t \geq-\tau$ (see, e.g., [10], [12], [14], and [24] ), which is denoted by $x\left(t ; x_{0}, r(0)\right)$ or $x\left(t ; \xi, r_{0}\right)$ in this note. It should be noted that (1) is a very general type of equation and includes stochastic differential equations, stochastic delay differential equations, integro-differential equations and those with Markovian switching. Much more equations are also included in (1) (see, e.g., [5] and [23]).

Let $C^{2,1}\left(R^{n} \times R_{+} \times S ; R_{+}\right)$denote the family of all nonnegative functions $V(x, t, i)$ on $R^{n} \times R_{+} \times S$ that are twice continuously differ- 
entiable in $x$ and once in $t$. If $V \in C^{2,1}\left(R^{n} \times R_{+} \times S ; R_{+}\right)$, define an operator associated with system $(1), \mathcal{L}$, from $C\left([-\tau, 0] ; R^{n}\right) \times R_{+} \times S$ to $R$ by

$$
\begin{aligned}
\mathcal{L} V\left(x_{t}, t, i\right) \\
=V_{t}(x, t, i)+V_{x}(x, t, i) f\left(x_{t}, t, i, u_{d}\right) \\
+\frac{1}{2} \text { trace } \\
\quad \times\left[g^{T}\left(x_{t}, t, i, u_{d}\right) V_{x x}(x, t, i) g\left(x_{t}, t, i, u_{d}\right)\right] \\
+\sum_{j=1}^{N} \gamma_{i j} V(x, t, j)
\end{aligned}
$$

where

$$
\begin{aligned}
V_{t}(x, t, i) & =\frac{\partial V(x, t, i)}{\partial t} \\
V_{x}(x, t, i) & =\left(\frac{\partial V(x, t, i)}{\partial x_{1}}, \ldots, \frac{\partial V(x, t, i)}{\partial x_{n}}\right) \\
V_{x x}(x, t, i) & =\left(\frac{\partial^{2} V(x, t, i)}{\partial x_{i} \partial x_{j}}\right)_{n \times n} .
\end{aligned}
$$

The purpose of this note is to develop the Razumihkin-type theorem on $p$ th moment input-to-state stability (ISS) of HSRSs and its applications. For definitions of $p$ th moment stability and input-to-state stability, readers are referred to, e.g., [6], [7], [9], [11], [17], [19], [20], and [21]. Let us introduce the definition of $p$ th moment ISS of HSRSs, which is consistent with the definition of ISS for deterministic systems (see, e.g., [9], [17], and [19]-[22]).

Definition 2.1: The system (1) is said to be $p$ th $(p>0)$ moment input-to-state stable (ISS) if there exist $\beta \in \mathcal{K} \mathcal{L}$ and $\gamma \in \mathcal{K}$ such that the solution $x(t)=x\left(t ; \xi, r_{0}\right)$ satisfies

$$
\mathbb{E}|x(t)|^{p} \leq \beta\left(\mathbb{E}\|\xi\|^{p}, t\right)+\gamma\left(\left\|u_{d}\right\|_{\infty}\right) \quad \forall t \geq 0
$$

for any essentially bounded input $u_{d} \in \mathcal{L}_{\infty}^{l}$ and any initial data $\xi \in$ $C_{\mathcal{F}_{0}}^{b}\left([-\tau, 0] ; R^{n}\right), r_{0} \in S$.

\section{RAZUMIKHIN-TYPE THEOREM ON ISS OF HSRSS}

As the main result of this note, we present a Razumikhin-type theorem on $p$ th moment ISS of HSRSs (1) as follows.

Theorem 3.1: Let $p>0, u \in V \mathcal{K}_{\infty}, v \in \mathcal{K}_{\infty}$ and $\lambda \in \mathcal{K}$. Assume that there exists a function $V \in C^{2,1}\left(R^{n} \times R_{+} \times S ; R_{+}\right)$such that

$$
\begin{aligned}
u\left(|x|^{p}\right) \leq V(x, t, i) & \\
& \leq v\left(|x|^{p}\right), \quad \forall(x, t, i) \in R^{n} \times[-\tau, \infty) \times S
\end{aligned}
$$

and, moreover, for all $1 \leq i \leq N$,

$$
\mathbb{E} \mathcal{L} V(\phi, t, i) \leq \lambda\left(\left|u_{d}(t)\right|\right)-\mathbb{E} w(\phi(0), i)
$$

for all $t \geq 0$ and those $\phi \in L_{\mathcal{F}_{t}}^{p}\left([-\tau, 0] ; R^{n}\right)$ satisfying

$$
\min _{k \in S} \mathbb{E} V(\phi(\theta), t+\theta, k)<\mathbb{E} q(\phi(0), t, i)
$$

on $-\tau \leq \theta \leq 0$, where $w: R^{n} \times S \rightarrow R_{+}$is a nonnegative function such that there is $\bar{w} \in \mathcal{K}_{\infty}$ with $w(x, i) \geq \bar{w}(|x|)$ and $\lim _{|x| \rightarrow \infty} \bar{w}(|x|) / v\left(|x|^{p}\right)>0$ for all $i \in S ; q: R^{n} \times R_{+} \times S \rightarrow R$ is a function such that $q(x, t, i)-V(x, t, i) \geq \zeta(|x|)$ for all $(x, t, i) \in$ $R^{n} \times[-\tau, \infty) \times S$ with $\zeta \in \mathcal{K}_{\infty}$ and $\lim _{|x| \rightarrow \infty} \zeta(|x|) / v\left(|x|^{p}\right)>0$. Then system (1) is $p$ th moment ISS.
In order to prove this theorem, let us present the following useful lemmas.

Lemma 3.1: Let $V(t)=V(x(t), t, r(t))$ for $t \geq 0$, then $\mathbb{E} V(t)$ is continuous on $t \geq 0$.

Proof: For any initial data $\xi \in C_{\mathcal{F}_{0}}^{b}\left([-\tau, 0] ; R^{n}\right)$, write $x(t)=$ $x(t ; \xi)$ and extend $r(t)$ to $[-\tau, 0)$ by setting $r(t)=r(0)=r_{0}$ for all $t \in[-\tau, 0)$. For convenience of the readers, the generalized Itô's formula is cited as follows (see [18] and [24])

$$
\begin{aligned}
& V(x(t), t, r(t)) \\
& =V(x(0), 0, r(0))+\int_{0}^{t} \mathcal{L} V\left(x_{s}, s, r(s)\right) \mathrm{d} s \\
& \quad+\int_{0}^{t} V_{x}(x(s), s, r(s)) g\left(x_{s}, s, r(s)\right) \mathrm{d} B(s) \\
& +\int_{0}^{t} \int_{R}[V(x(s), s, r(0)+h(r(s), l)) \\
& \quad-V(x(s), s, r(s))] \mu(\mathrm{d} s, \mathrm{~d} l)
\end{aligned}
$$

for all $t \geq 0$, where function $h(\cdot, \cdot)$ and martingale measure $\mu(\cdot, \cdot)$ are defined as, e.g., (2.6) and (2.7) in [24] (see also [4] and [2]).

Since $\xi \in C_{\mathcal{F}_{0}}^{b}\left([-\tau, 0] ; R^{n}\right)$, we can find an integer $k_{0}$ such that $\|\xi\|<k_{0}$ a.s.. For any integer $k>k_{0}$, define the stopping time

$$
\rho_{k}=\inf \{t \geq 0:|x(t)| \geq k\}
$$

where we set $\inf \emptyset=\infty$ as usual. Note that $x(t)$ is continuous and so are $|x(t)|$ and $v(|x(t)|)$ on $t \geq-\tau$. Clearly, $\rho_{k} \rightarrow \infty$ almost surely as $k \rightarrow \infty$. Moreover, since $x_{0}=\xi \in C_{\mathcal{F}_{0}}^{b}\left([-\tau, 0] ; R^{n}\right)$, $\mathbb{E} V(x(0), 0, r(0)) \leq \mathbb{E} v(|\xi(0)|) \leq v\left(k_{0}\right)$. It then follows from (6) that

$$
\begin{aligned}
& \mathbb{E} V\left(x\left(t_{k}\right), t_{k}, r\left(t_{k}\right)\right) \\
& \quad=\mathbb{E} V(x(0), 0, r(0))+\mathbb{E} \int_{0}^{t_{k}} \mathcal{L} V\left(x_{s}, s, r(s)\right) \mathrm{d} s
\end{aligned}
$$

where $t_{k}=t \wedge \rho_{k}$. So, letting $k \rightarrow \infty$, by Fubini's theorem, we have

$$
\begin{aligned}
\mathbb{E} V(t) & =\mathbb{E} V(0)+\mathbb{E} \int_{0}^{t} \mathcal{L} V\left(x_{s}, s, r(s)\right) \mathrm{d} s \\
& =\mathbb{E} V(0)+\int_{0}^{t} \mathbb{E} \mathcal{L} V\left(x_{s}, s, r(s)\right) \mathrm{d} s
\end{aligned}
$$

for all $t \geq 0$. This implies $\mathbb{E} V(t)$ is continuous on $t \geq 0$.

Lemma 3.2: For any $t \geq 0$, there is $a_{w}>0$ such that $\mathbb{E} w(x, i) \geq$ $a_{w}$ for all $i \in S$ whenever $\mathbb{E} V(x, t, i) \geq a_{v}>0$.

Proof: It immediately follows the desired conclusion if we show there is $\mu_{w} \in \mathcal{K}_{\infty}$ such that

$$
\mathbb{E} \bar{w}(|x(t)|) \geq \mu_{w}\left(a_{v}\right)
$$

whenever $\mathbb{E} v\left(|x|^{p}\right) \geq \mathbb{E} V(x, t, i) \geq a_{v}>0$.

Fix $t$ for the moment. We define a nondecreasing function $b: R_{+} \rightarrow$ $R_{+}$as

$$
b(y)=\inf _{|x|^{p} \geq v^{-1}(y / 2)} \frac{\bar{w}(|x|)}{v\left(|x|^{p}\right)}, \quad y \geq 0 .
$$


By property of function $\bar{w}(\cdot), b(y)>0$ when $y>0$. So, for any $a_{v}>0$, we have

$$
\begin{aligned}
\mathbb{E} \bar{w}(|x|) & \geq \int_{|x|^{p} \geq v-1\left(a_{v} / 2\right)} \bar{w}(|x|) \mathrm{d} \mathbb{P} \\
& \geq b\left(a_{v}\right) \int_{v\left(|x|^{p}\right) \geq a_{v} / 2} v\left(|x|^{p}\right) \mathrm{d} \mathbb{P} \geq \frac{a_{v} b\left(a_{v}\right)}{2}
\end{aligned}
$$

whenever $\mathbb{E} v\left(|x|^{p}\right) \geq \mathbb{E} V(x, t, i) \geq a_{v}$. Inequality (11) holds with $\mu_{w}\left(a_{v}\right)=a_{v} b\left(a_{v}\right) / 2$.

Lemma 3.3: For any $t \geq 0$, there is $a_{q}>0$ such that $\mathbb{E} q(x, t, i) \geq$ $a_{q}+\mathbb{E} V(x, t, i)$ for all $i \in S$ whenever $\mathbb{E} V(x, t, i) \geq a_{v}>0$.

Proof: It is noted that $\mathbb{E} q(x, t, i)-\mathbb{E} V(x, t, i) \geq \mathbb{E} \zeta(|x|)$ for all $t \geq 0$. According to the property of function $\zeta(|x|)$, the rest of the proof is similar to that of Lemma 3.2 and hence omitted.

We can now begin to prove Theorem 3.1.

Proof: Denote $\alpha_{\lambda}=\lambda\left(\left\|u_{d}\right\|_{\infty}\right)$ and $\bar{V}_{0}=u\left(\mathbb{E}\|\xi\|^{p}\right)$. Without loss of generality, assume $0<\mu_{w}^{-1}\left(2 \alpha_{\lambda}\right)<$ $u\left(\sup _{-\tau<\theta<0} \mathbb{E}|\xi(\theta)|^{p}\right) \leq \bar{V}_{0}$. For any $t \geq 0$, by Lemma 3.2, $\mathbb{E} w(x(t), i) \geq 2 \alpha_{\lambda}$ whenever $\mathbb{E} V(x, t, i) \geq \mu_{w}^{-1}\left(2 \alpha_{\lambda}\right)$ for all $i \in S$. By Lemma 3.3, there is $a>0$ such that $\mathbb{E} q(x, t, i)-\mathbb{E} V(x, t, i) \geq a$, $i \in S$, whenever $\mathbb{E} V(x, t, i) \geq \mu_{w}^{-1}\left(2 \alpha_{\lambda}\right)$. Let $J$ be the minimal nonnegative integer such that $M_{0}=\mu_{w}^{-1}\left(2 \alpha_{\lambda}\right)+J a>\bar{V}_{0}$. Moreover, let $\tilde{\tau}=\tau \vee M_{0} / \alpha_{\lambda}$ and $t_{j}=j \tilde{\tau}$ for $j=0,1,2, \ldots, J$. We claim that

$$
\mathbb{E} V(x(t), t, r(t)) \leq \bar{V}_{0} \wedge M_{j}
$$

for all $t \geq t_{j}$, where $M_{j}=\mu_{w}^{-1}\left(2 \alpha_{\lambda}\right)+(J-j) a$ and $j=0,1,2, \ldots, J$.

First we show that

$$
\mathbb{E} V(x(t), t, r(t)) \leq \bar{V}_{0}, \quad \forall t \geq t_{0} .
$$

Suppose that $t_{a}=\inf \left\{t>t_{0}: \mathbb{E} V(x(t), t, r(t))>\bar{V}_{0}\right\}<\infty$. Since $\mathbb{E} V(x(t), t, r(t))$ is continuous on $t \geq 0$, there exist a pair of constants $t_{b}$ and $t_{c}$ such that $t_{0} \leq t_{b} \leq t_{a}<t_{c}$ and

$$
\begin{cases}\mathbb{E} V(x(t), t, r(t))=\bar{V}_{0}, & t=t_{b} ; \\ \bar{V}_{0}<\mathbb{E} V(x(t), t, r(t))<\bar{V}_{0}+a, & t_{b}<t \leq t_{c} .\end{cases}
$$

However, by (10) and condition (5), we have

$$
\begin{aligned}
\mathbb{E} V(x(t), t, r(t))= & \mathbb{E} V\left(x\left(t_{b}\right), t_{b}, r\left(t_{b}\right)\right) \\
& +\int_{t_{b}}^{t} \mathbb{E} \mathcal{L} V\left(x_{s}, s, r(s)\right) \mathrm{d} s \\
\leq & \bar{V}_{0}-\alpha_{\lambda}\left(t-t_{b}\right)<\bar{V}_{0}
\end{aligned}
$$

for every $t \in\left(t_{b}, t_{c}\right]$, which contradicts (15). So inequality (14) must be true.

We further show that $\mathbb{E} V(x(t), t, r(t)) \leq M_{1}$ for all $t \geq t_{1}$. Let $\tau_{1}=\inf \left\{t \geq t_{0}: \mathbb{E} V(x(t), t, r(t)) \leq M_{1}\right\}$. If $\tau_{1}>t_{1}$, then, $\forall t_{0} \leq t \leq t_{1}$, we have

$$
\begin{aligned}
\mathbb{E} q & (x(t), t, r(t)) \\
& \geq \mathbb{E} V(x(t), t, r(t))+a>M_{1}+a>\bar{V}_{0} \\
& \geq \mathbb{E} V(x(t+\theta), t+\theta, r(t+\theta)) \\
& \geq \min _{k \in S} \mathbb{E} V(\phi(\theta), t+\theta, k), \quad \forall \theta \in[-\tau, 0] .
\end{aligned}
$$

This, by condition (5), implies $\mathbb{E} \mathcal{L} V\left(x_{t}, t, r(t)\right) \leq-\alpha_{\lambda}$ a.e. on $\left[t_{0}, t_{1}\right]$. Consequently, by (10), we have $\mathbb{E} V\left(x\left(t_{1}\right), t_{1}, r\left(t_{1}\right)\right) \leq$ $\bar{V}_{0}-\alpha_{\lambda} \tilde{\tau}<0$, which contradicts the property of $\mathbb{E} V(x(t), t, r(t)) \geq 0$ for all $t \geq 0$. So we must have $\tau_{1} \leq t_{1}$. Let $t_{1 a}=\inf \left\{t>\tau_{1}\right.$ :
$\left.\mathbb{E} V(x(t), t, r(t))>M_{1}\right\}$. If $t_{1 a}<\infty$, then there are constants $t_{1 b}$ and $t_{1 c}$ such that $t_{1} \leq t_{1 b} \leq t_{1 a}<t_{1 c}$ and

$$
\begin{cases}\mathbb{E} V(x(t), t, r(t))=M_{1}, & t=t_{1 b} ; \\ M_{1}<\mathbb{E} V(x(t), t, r(t))<M_{1}+a, & t_{1 b}<t \leq t_{1 c} .\end{cases}
$$

Similarly, by (10) and (5), we find a contradiction and hence have (13) for $j=1$.

Define $\tau_{j}=\inf \left\{t \geq t_{j-1}: \mathbb{E} V(x(t), t, r(t)) \leq M_{j}\right\}$ for $j=2,3, \ldots, J$. By the same type of reasoning, we have $\mathbb{E} V(x(t), t, r(t)) \leq M_{j}$ for all $t \geq t_{j}$ and $j=2,3, \ldots, J$. Particularly, $\mathbb{E} V(x(t), t, r(t)) \leq M_{J}=\mu_{w}^{-1}\left(2 \alpha_{\lambda}\right)$ for all $t \geq t_{J}$. By Jensen's inequality, we have

$$
\mathbb{E}|x(t)|^{p} \leq \gamma\left(\left\|u_{d}\right\|_{\infty}\right), \quad \forall t \geq t_{J}
$$

where $\gamma(\cdot)=u^{-1}\left(\mu_{w}^{-1}(2 \lambda(\cdot))\right)$.

Let $k=\bar{V}_{0} / t_{J}$. Choose $\tilde{\beta} \in \mathcal{K} \mathcal{L}$ such that $\tilde{\beta}\left(\bar{V}_{0}, t\right) \geq 2 \bar{V}_{0}-k t$ for all $0 \leq t \leq t_{J}$. So we have $\mathbb{E} V(x(t), t, r(t)) \leq \tilde{\beta}\left(\bar{V}_{0}, t\right)$ for all $0 \leq t \leq t_{J}$, which implies

$$
\mathbb{E}|x(t)|^{p} \leq u^{-1}\left(\tilde{\beta}\left(\bar{V}_{0}, t\right)\right)=\beta\left(\mathbb{E}\|\xi\|^{p}, t\right), \quad \forall 0 \leq t \leq t_{J}
$$

where $\beta(\cdot, \cdot)=u^{-1}(\tilde{\beta}(u(\cdot), \cdot))$ is a $\mathcal{K} \mathcal{L}$ function. This completes the proof.

Remark 3.1: Obviously, inequality (3) implies that system (1) with $u_{d}(t) \equiv 0$ is globally $p$ th moment asymptotically stable. Moreover, it is not difficult to show that if $|u(t)| \rightarrow 0$ as $t \rightarrow \infty$, so does $\mathbb{E}|x(t)|^{p}$ (see, e.g. [9, Exercise 4.58]). Therefore, by Theorem 3.1, it is easy to find that the HSDS, considered in [24, Example 2.1] but with modedependent and time-varying delay $\tilde{\tau}: R_{+} \times S \rightarrow[0, \tau]$, is mean-quare asymptotically stable while the results in [24] do not work.

Remark 3.2: It is noted that inequality (6) removes the maximum operator on the right-hand side of corresponding conditions in the existing results (see[15, Theorem 2.1] and [7, Theorem 3.2]), which makes Theorem 3.1 less conservative but more applicable (see Example 4.1).

\section{APPLICATION AND EXAMPLE}

Hybrid stochastic delay systems (HSDSs) described with stochastic differential delay equations with Markovian switching are an important class of HSRSs that are frequently used in engineering. As an illustrative example of applications of our new result, we consider the following HSDE

$$
\begin{aligned}
\mathrm{d} x(t)=F( & \left.x(t), x(t-\delta(t, r(t))), t, r(t), u_{d}(t)\right) \mathrm{d} t \\
& +G\left(x(t), x(t-\delta(t, r(t))), t, r(t), u_{d}(t)\right) \mathrm{d} B(t)
\end{aligned}
$$

on $t \geq 0$, where $\delta: R_{+} \times S \rightarrow[0, \tau]$ is Borel measurable while $F: R^{n} \times R^{n} \times R_{+} \times S \times R^{l} \rightarrow R^{n}$ and $G: R^{n} \times R^{n} \times R_{+} \times S \times R^{l} \rightarrow R^{n \times m}$ are measurable functions with $F(0,0, t, i, 0) \equiv 0$ and $g(0,0, t, i, 0) \equiv 0$ for all $t \geq 0$ and $i \in S$. Actually, this is a special case of (1) when $f\left(\phi, t, i, u_{d}\right)=F\left(\phi(0), \phi(-\delta(t, i)), t, i, u_{d}\right)$ and $g\left(\phi, t, i, u_{d}\right)=G\left(\phi(0), \phi(-\delta(t, i)), t, i, u_{d}\right)$ for $(\phi, t, i) \in$ $C\left([-\tau, 0] ; R^{n}\right) \times R_{+} \times S \times R^{l}$ while the operator $\mathcal{L}$ defined in (2) becomes from $R^{n} \times R^{n} \times R_{+} \times S$ to $R$ as

$$
\begin{aligned}
\mathcal{L} V & (x, y, t, i) \\
= & V_{t}(x, t, i)+V_{x}(x, t, i) F\left(x, y, t, i, u_{d}\right) \\
& +\frac{1}{2} \operatorname{trace}\left[G^{T}\left(x, y, t, i, u_{d}\right) V_{x x}(x, t, i) G\left(x, y, t, i, u_{d}\right)\right] \\
& +\sum_{j=1}^{N} \gamma_{i j} V(x, t, j) .
\end{aligned}
$$


Let us use Theorem 3.1 to establish a useful criterion for system (19).

Theorem 4.1: Let $p>0, u \in V \mathcal{K}_{\infty}, v \in \mathcal{K}_{\infty}, \lambda \in \mathcal{K}$ and $\kappa_{0 i} \geq$ $\kappa_{1 i} \geq 0, i \in S$. Assume that there exists a function $V \in C^{2,1}\left(R^{n} \times\right.$ $R_{+} \times S ; R_{+}$) such that inequality (4) holds and, moreover,

$$
\begin{aligned}
\mathcal{L} V(x, y, t, i) \leq \lambda\left(\left|u_{d}(t)\right|\right)- & \hat{\zeta}(x, i)-\kappa_{0 i} V(x, t, i) \\
& +\kappa_{1 i} \min _{1 \leq k \leq N} V(y, t-\delta(t, i), k)
\end{aligned}
$$

for all $(x, y, t, i) \in R^{n} \times R^{n} \times R_{+} \times S$, where $\hat{\zeta}: R^{n} \times S \rightarrow R$ is a function such that there is $\hat{w} \in \mathcal{K}$ with $\hat{\zeta}(x, i) \geq \hat{w}(|x|)$ for all $i \in S$ and $\lim _{|x| \rightarrow \infty} \hat{w}(|x|) / v\left(|x|^{p}\right)>0$. Then system (19) is $p$ th moment ISS.

Proof: For any $i \in S$, let

$$
\begin{aligned}
w(x, i) & =\frac{1}{1+\kappa_{0 i}} \hat{\zeta}(x, i) \quad \text { and } \\
q(x, t, i) & =V(x, t, i)+w(x, i)
\end{aligned}
$$

in inequalities (5) and (6). By inequality (21) and Fatou's lemma, we have

$$
\begin{aligned}
\mathbb{E} \mathcal{L} V & (x, y, t, i) \\
\leq & \lambda\left(\left|u_{d}(t)\right|\right)-\mathbb{E} \hat{\zeta}(x, i)-\kappa_{0 i} \mathbb{E} V(x, t, i) \\
& +\kappa_{1 i} \mathbb{E}\left[\min _{1 \leq k \leq N} V(y, t-\delta(t, i), k)\right] \\
\leq & \lambda\left(\left|u_{d}(t)\right|\right)-\kappa_{0 i}[\mathbb{E} V(x, t, i)+\mathbb{E} w(x, i)] \\
& +\kappa_{1 i} \min _{1 \leq k \leq N} \mathbb{E} V(y, t-\delta(t, i), k) \\
& -\mathbb{E} w(x, i) \\
\leq & \lambda\left(\left|u_{d}(t)\right|\right)-\mathbb{E} w(x, i) \\
& -\left(\kappa_{0 i}-\kappa_{1 i}\right)[\mathbb{E} V(x, t, i)+\mathbb{E} w(x, i)] \\
\leq & \lambda\left(\left|u_{d}(t)\right|\right)-\mathbb{E} w(x, i)
\end{aligned}
$$

for all $t \geq 0, i \in S$ and $x_{t} \in L_{\mathcal{F}_{t}}^{p}\left([-\tau, 0] ; R^{n}\right)$ satisfying condition (6) with function $q(x, t, i)$ defined in (22), i.e., $\min _{k \in S} \mathbb{E} V(y, t-$ $\delta(t, i), k)<\mathbb{E} V(x, t, i)+\mathbb{E} w(x, i)$. Moreover, $\bar{w}(\cdot)=\zeta(\cdot)=$ $\hat{w}(\cdot) / 1+\kappa$ satisfy the properties required in (5) and (6). By Theorem 3.1 , inequality (3) holds for system (19).

To compare with the existing result in [7], let us consider the following example.

Example 4.1: Let $B(t)$ be a scalar Brownian motion. Let $r(t)$ be a right-continuous Markovian chain independent of $B(t)$ and taking values in $S=\{1,2\}$ with generator

$$
\Gamma=\left(\gamma_{i j}\right)_{2 \times 2}=\left(\begin{array}{cc}
-1 & 1 \\
2 & -2
\end{array}\right) .
$$

Consider a scalar uncertain stochastic delay system with Markovian switching of the form

$\mathrm{d} x(t)=f(x(t), t, r(t)) \mathrm{d} t+g(x(t-\delta(t, r(t))), t, r(t)) \mathrm{d} B(t)$

on $t \geq 0$, where $\delta: R_{+} \times S \rightarrow[-\tau, 0]$ is a continuous but nondifferentiable function with respect to $t$ and

$$
\begin{aligned}
& f(x, t, 1)=\frac{1}{4} x-\frac{1}{8}|x| \sqrt{x} \\
& f(x, t, 2)=-b x-\frac{1}{10} x^{3} \\
& g(y, t, 1)=\frac{1}{4} y \cos t, \quad g(y, t, 2)=\sqrt{2} y \sin t .
\end{aligned}
$$

with $x=x(t), y=x(t-\delta(t, r(t)))$ and positive constant $b$.
It is noted that the existing results [14], [15], [24], [25] can not be applied to system (23), which has mode-dependent and time-varying delay $\delta(t, r(t))$. Observe that

and

$$
\left\{\begin{array}{l}
2 x f(x, t, 1) \leq \frac{1}{2} x^{2}-\frac{1}{4}|x|^{7 / 3} \\
g^{2}(y, t, 1) \leq \frac{1}{16} y^{2}
\end{array}\right.
$$

$$
\left\{\begin{array}{l}
2 x f(x, t, 2) \leq-2 b x^{2}-\frac{1}{5} x^{4} \\
g^{2}(y, t, 2) \leq 2 y^{2} .
\end{array}\right.
$$

To examine the stability of system (23), we construct a Lyapunov function candidate $V: R \times S \rightarrow R_{+}$as $V(x, i)=\alpha_{i} x^{2}$ with $\alpha_{2}=1$ and $\alpha_{1}>0$ to be determined. By computation, we have

$$
\begin{aligned}
\mathcal{L} V(x, y, t, 1) \leq & -\frac{\alpha_{1}}{4}|x|^{7 / 3} \\
& -\left(\frac{\alpha_{1}}{2}-1\right) x^{2}+\frac{\alpha_{1}}{16} y^{2} \\
\mathcal{L} V(x, y, t, 2) \leq & -\frac{1}{5} x^{4} \\
& -\left(2+2 b-2 \alpha_{1}\right) x^{2}+2 y^{2} .
\end{aligned}
$$

According to Theorem 4.2 in [7], inequalities (24) and (25) give

$$
\begin{aligned}
& \lambda_{01}=\frac{1}{2}-\frac{1}{\alpha_{1}}, \quad \lambda_{11}=\frac{\alpha_{1}}{16}, \quad \lambda(s, 1)=\frac{1}{4 \sqrt{\alpha_{1}}} s^{7 / 6} ; \\
& \lambda_{02}=\frac{2(1+b)}{\alpha_{1}}-2, \quad \lambda_{12}=2, \quad \lambda(s, 2)=\frac{1}{5 \alpha_{1}^{2}} s^{2} .
\end{aligned}
$$

Inequalities $\lambda_{01} \geq \lambda_{11}$ and $\lambda_{02} \geq \lambda_{12}$ yield $\alpha_{1}=4$ and $b \geq 7$. Then, by Theorem 4.2 in [7], system (23) is mean-square asymptotically stable if $b \geq 7$. However, for inequalities (24) and (25), we have

$$
\begin{aligned}
& \kappa_{01}=\frac{1}{2}-\frac{1}{\alpha_{1}}, \quad \kappa_{11}=\frac{\alpha_{1}}{16}, \quad \hat{\zeta}(x, 1)=\frac{\alpha_{1}}{4}|x|^{7 / 3} ; \\
& \kappa_{02}=2\left(1+b-\alpha_{1}\right), \quad \kappa_{12}=2, \quad \hat{\zeta}(x, 2)=\frac{1}{5} x^{4} .
\end{aligned}
$$

Inequalities $\kappa_{01} \geq \kappa_{11}$ and $\kappa_{02} \geq \kappa_{12}$ imply $\alpha_{1}=4$ and $b \geq 4$. By Theorem 4.1, the sufficient condition for mean-square asymptotic stability of system (23) is $b \geq 4$. Note that, when $4 \leq b<7$, Theorem 4.2 in [7] does not work while Theorem 4.1 is still applicable to system (23). This shows Theorem 4.1 is more applicable.

\section{CONCLUSION}

This note improves an existing result in [7] and develops a Razumikhin-type theorem on input-to-state stability of HSRSs in $p$ th $(p>$ 0 ) moment sense. It is seen that this improved result is less conservative but more applicable (see Remark 3.1, Remark 3.2 and Example 4.1).

\section{ACKNOWLEDGMENT}

The authors gratefully acknowledge the reviewers and the associate editor's comments.

\section{REFERENCES}

[1] M. Athens, "Command and control (C2) theory: A challenge to control science," IEEE Trans. Automat. Control, vol. AC-32, no. 4, pp. 286-293, Apr. 1987.

[2] G. K. Basak, A. Bisi, and M. K. Ghosh, "Stability of a random diffusion with linear drift," J. Math. Anal. Appl., vol. 202, pp. 604-622, 1996.

[3] E. K. Bouka, Z. K. Liu, and G. X. Liu, "Delay-dependent robust stability and $H_{\infty}$ control of jump linear systems with time-delay," Int. J. Control, vol. 74, pp. 329-340, 2001.

[4] M. K. Ghosh, A. Arapostathis, and S. I. Marcus, "Optimal control of switching diffusions with application to flexible manufacturing systems," SIAM J. Control Optim., vol. 31, pp. 1183-1204, 1993. 
[5] J. K. Hale and S. M. V. Lunel, Introduction to Functional Differential Equations. New York: Springer-Verlag, 1993.

[6] L. Huang and F. Deng, "Razumikhin-type theorems on stability of neutral stochastic functional differential equations," IEEE Trans. Automat. Control, vol. 53, no. 7, pp. 1718-1723, Aug. 2008.

[7] L. Huang, X. Mao, and F. Deng, "Stability of hybrid stochastic retarded systems," IEEE Trans. Circuits Syst. I: Regular Papers, vol. 55, no. 11, pp. 3413-3420, Dec. 2008.

[8] Y. Ji and H. J. Chizeck, "Controllability, stabilizability and continuoustime Markovian jump linear quadratic control," IEEE Trans. Automat. Control, vol. 35, no. 7, pp. 777-788, Jul. 1990.

[9] H. K. Khalil, Nonlinear Systems, 3rd ed. Englewood Cliffs, NJ: Prentice Hall, 2002.

[10] S.-J. Liu, S. S. Ge, and J.-F. Zhang, "Adaptive output-feedback control for a class of uncertain stochastic non-linear systems with time delays," Int. J. Control, vol. 81, pp. 1210-1220, 2008.

[11] S.-J. Liu, Z.-P. Jiang, and J.-F. Zhang, "Global output-feedback stabilization for a class of stochastic non-minimum-phase nonlinear systems," Automatica, vol. 44, pp. 1944-1957, 2008.

[12] X. Mao, "Razumikhin-type theorems on exponential stability of stochastic functional differential equations," Stochastic Processes Appl., vol. 65 , pp. 233-250, 1996

[13] X. Mao, A. Matasov, and A. B. Piunovskiy, "Stochastic differential delay equations with Markovian switching," in Proc. Bernoulli Conf., 2000, vol. 6, pp. 73-90.

[14] X. Mao, "Exponential stability of stochastic delay interval systems with Markovian switching," IEEE Trans. Automat. Control, vol. 47, no. 10, pp. 1604-1612, Oct. 2002.

[15] X. Mao, J. Lam, S. Xu, and H. Gao, "Razumikhin method and exponential stability of hybrid stochastic delay interval systems," J. Math. Anal. Appl., vol. 314, pp. 45-66, 2006.

[16] M. Mariton, Jump Linear Systems in Automatic Control. New York: Marcel Dekker, 1990.

[17] P. Pepe and Z.-P. Jiang, "A Lyapunov-Krasovskii methodology for ISS and iISS of time-delay systems," Syst. Control Lett., vol. 55, pp. 1006-1014, 2006

[18] A. V. Skorohod, Asymptotic Methods in the Theory of Stochastic Differential Equations. Providence, RI: American Mathematical Society, 1989

[19] E. D. Sontag, "Smooth stabilization implies coprime factorization," IEEE Trans. Automat. Control, vol. AC-34, no. 4, pp. 435-443, Apr. 1989.

[20] E. D. Sontag and Y. Wang, "New characterizations of input-to-state stability," IEEE Trans. Automat. Control, vol. AC-41, no. 9, pp. 1283-1294, Sep. 1996.

[21] D. D. Sworder and R. O. Rogers, "An LQ-solution to a control problem associated with a solar thermal central receiver," IEEE Trans. Automat. Control, vol. AC-28, no. 10, pp. 971-978, Oct. 1983.

[22] A. R. Teel, "Connection between Razumikhin-type theorems and the ISS nonlinear small gain theorem," IEEE Trans. Automat. Control, vol. 43, no. 7, pp. 960-964, Jul. 1998.

[23] B. Xu, "Stability of retarded dynamic systems: A Lyapunov function approach," J. Math. Anal. Appl., vol. 253, pp. 590-615, 2001.

[24] C. Yuan and X. Mao, "Robust stability and controllability of stochastic differential delay equations with Markovian switching," Automatica, vol. 40, pp. 343-354, 2004

[25] D. Yue and Q.-L. Han, "Delay-dependent exponential stability of stochastic systems with time-varying delay, nonlinearity, and Markovian switching," IEEE Trans. Automat. Control, vol. 50, no. 2, pp. 217-222, Feb. 2005.

\section{Signal-to-Noise Ratio Fundamental Limitations in Continuous-Time Linear Output Feedback Control}

\author{
Alejandro J. Rojas, Member, IEEE
}

Abstract-In the present technical note we study the fundamental limitation on stability that arise when an additive coloured Gaussian noise (ACGN) channel is explicitly considered over either the control or measurement paths of a linear time invariant (LTI) feedback loop. By considering a linear setting we can naturally express the fundamental limitation as a lower bound on the channel signal-to-noise ratio (SNR) required for stabilisability. We start by first obtaining a closed-form expression for the squared $L_{2}$ norm of a partial fraction expansion with repeated poles in the Laplace domain. We then use the squared $L_{2}$ norm result to obtain the closed-form expression for the infimal SNR required for stabilisability. The proposed closed-form includes the case of repeated unstable plant poles and non minimum phase (NMP) zeros.

Index Terms-Additive coloured Gaussian noise (ACGN), signal-to-noise ratio (SNR).

\section{INTRODUCTION}

Fundamental limitations in control design as been an important area of research with early seminal results such as [1] and [2]. For a linear time invariant (LTI) plant it is well understood how unstable poles, non minimum phase (NMP) zeros and time-delay cause unavoidable limitations both in regulation and performance (see for example [3] and references therein). In recent years, the study of fundamental limitations has been extended to problems of control over communication networks, [4, Theorem 4.6], [5], attracting a growing interest (see for example [6] and the recent survey by [7]).

Most results in control over network use information theoretic arguments to obtain necessary and sufficient lower bounds on, for example, the transmission data rate for noiseless channels [8], [9] or noisy channels [10]-[13] required for stabilisability. In particular, for linear plant models, in [12, Proposition III.1], it has been shown that if the unstable plant is to be stabilised, then the capacity of a noisy channel in the control loop has to satisfy a lower bound that depends on the unstable eigenvalues of the plant. A similar result was proved earlier in $[8$, Theorem 2.1], for the transmission data rate of a noiseless channel and further extended to nonlinear plant models in [9, Theorem 1].

Another line of research, that does not center on channel capacity nor transmission rate, introduces a framework to study stabilisability of a feedback loop over channels that have a signal to noise ratio (SNR) constraint [14], [15] (and related work in [16], [17]). A recognisable characteristic of the proposed SNR approach is that it is a linear formulation allowing the use of all the linear design techniques (such as cheap control for example). The authors of [14], [18] obtained the expression for the infimal SNR required to stabilise a finite dimensional unstable LTI plant over a memoryless additive white Gaussian noise (AWGN) channel when considering unstable plant poles, NMP zeros and plant time-delay. In [15] the infimal SNR is computed for the case of additive coloured Gaussian noise (ACGN) channels with bandwidth limitation, see Fig. 1. The bandwidth limitation may be imposed for several reasons, for example to avoid interference between different channels in a communication system or to model communications hardware proper-

Manuscript received May 14, 2008; revised December 08, 2008. First published July 24, 2009; current version published August 05, 2009. Recommended by Associate Editor M. Fujita.

The author is with the ARC Centre of Excellence for Complex Dynamic Systems and Control, The University of Newcastle, Newcastle 2308, Australia (e-mail: alejandro.rojas@newcastle.edu.au).

Digital Object Identifier 10.1109/TAC.2009.2022114 